University of New Hampshire

University of New Hampshire Scholars' Repository

$11-1999$

\title{
The economic implications of HLA matching in cadaveric renal transplantation.
}

\author{
Mark A. Schnitzler \\ Washington University School of Medicine in St. Louis \\ Christopher S. Hollenbeak \\ Washington University School of Medicine in St. Louis \\ David S. Cohen \\ Washington University School of Medicine in St. Louis \\ Robert Woodward \\ University of New Hampshire - Main Campus \\ Jeffrey A. Lowell \\ Washington University School of Medicine in St. Louis
}

See next page for additional authors

Follow this and additional works at: https://scholars.unh.edu/hmp_facpub

Part of the Medicine and Health Sciences Commons

\section{Recommended Citation}

Schnitzler, M.A., Hollenbeak, C.S., Cohen, D.S., Woodward, R.S., Lowell, J.A., Singer, G.G., Tesi, R.J., Howard, T.K., Mohanakumar, T., Brennan, D.C. The economic implications of HLA matching in cadaveric renal transplantation. (1999) New England Journal of Medicine, 341 (19), pp. 1440-1446.

This Article is brought to you for free and open access by the Health Management and Policy at University of New Hampshire Scholars' Repository. It has been accepted for inclusion in Health Management and Policy Scholarship by an authorized administrator of University of New Hampshire Scholars' Repository. For more information, please contact Scholarly.Communication@unh.edu. 


\section{Authors}

Mark A. Schnitzler, Christopher S. Hollenbeak, David S. Cohen, Robert Woodward, Jeffrey A. Lowell, Gary G. Singer, Raymond J. Tesi, Todd K. Howard, T Mohanakumar, and Daniel C. Brennan 


\section{THE ECONOMIC IMPLICATIONS OF HLA MATCHING IN CADAVERIC RENAL TRANSPLANTATION}

Mark A. Schnitzler, Ph.D., Christopher S. Hollenbeak, M.A., David S. Cohen, M.A., Robert S. Woodward, Ph.D., Jeffrey A. Lowell, M.D., Gary G. Singer, M.D., Raymond J. Tesi, M.D., Todd K. Howard, M.D.,

T. Mohanakumar, Ph.D., and Daniel C. Brennan, M.D.

\begin{abstract}
Background The potential economic effects of the allocation of cadaveric kidneys on the basis of tissue-matching criteria are controversial. We analyzed the economic costs associated with the transplantation of cadaveric kidneys with various numbers of HLA mismatches and examined the potential economic benefits of a local, as compared with a national, system designed to minimize HLA mismatches between donor and recipient in first cadaveric renal transplantations.
\end{abstract}

Methods All data were supplied by the U.S. Renal Data System. Data on all payments made by Medicare from 1991 through 1997 for the care of recipients of a first cadaveric renal transplant were analyzed according to the number of HLA-A, B, and DR mismatches between donor and recipient and the duration of cold ischemia before transplantation.

Results Average Medicare payments for renaltransplant recipients in the three years after transplantation increased from $\$ 60,436$ per patient for fully HLA-matched kidneys (those with no HLA-A, B, or DR mismatches) to $\$ 80,807$ for kidneys with six HLA mismatches between donor and recipient, a difference of 34 percent $(P<0.001)$. By three years after transplantation, the average Medicare payments were $\$ 64,119$ for transplantations of kidneys with less than 12 hours of cold-ischemia time and $\$ 74,997$ for those with more than 36 hours $(P<0.001)$. In simulations, the assignment of cadaveric kidneys to recipients by a method that minimized HLA mismatching within a local geographic area (i.e., within one of the approximately 50 organ-procurement organizations, which cover widely varying geographic areas) produced the largest cost savings $(\$ 4,290$ per patient over a period of three years) and the largest improvements in the graft-survival rate (2.3 percent) when the potential costs of longer cold-ischemia time were considered.

Conclusions Transplantation of better-matched cadaveric kidneys could have substantial economic advantages. In our simulations, HLA-based allocation of kidneys at the local level produced the largest estimated cost savings, when the duration of cold ischemia was taken into account. No additional savings were estimated to result from a national allocation program, because the additional costs of longer coldischemia time were greater than the advantages of optimizing HLA matching. (N Engl J Med 1999;341: 1440-6.)

(C)1999, Massachusetts Medical Society.

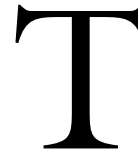

HE debate about the value of HLA matching in renal transplantation continues. Large retrospective studies have shown the potential for clear improvements in graft survival as a result of better HLA matching. ${ }^{1-4}$ A prospective study of mandatory national assignment of cadaveric kidneys to recipients with no HLA antigen mismatches demonstrated benefits in terms of graft survival. ${ }^{2}$ An expanded policy of assigning cadaveric kidneys to recipients with whom there are no HLA mismatches (i.e., no HLA-A, B, or DR antigen mismatches), regardless of geographic region, has been implemented, but further expansion of the requirement for national assignment of kidneys to recipients with whom there is some degree of HLA mismatching has met strong resistance.

The current allocation policy of the United Network for Organ Sharing (UNOS) favors better matching of kidneys and recipients with some degree of HLA incompatibility on the local level (i.e., within one of the approximately 50 organ-procurement organizations, which cover widely varying geographic areas), but it does not directly discourage transplantation when the most donor-recipient mismatches are present - that is, mismatches of five or six HLA antigens. Only a national system of allocation could minimize HLA mismatches in all cases. The current national scheme for assigning cadaveric kidneys to recipients with whom there are no HLA mismatches, regardless of the geographic region, increases the duration of cold ischemia (the time from donation to transplantation). Increasing cold-ischemia time might decrease graft survival and increase costs if a national allocation system were implemented. In addition, strategies to minimize HLA mismatching might further skew the racial distribution of transplants toward whites and away from members of minority groups, thus raising questions about the ethics of such a system. ${ }^{5}$

From Pharmaco-economic Transplant Research (M.A.S., R.S.W., J.A.L., T.K.H., D.C.B.), the Health Administration Program (M.A.S., C.S.H., D.S.C., R.S.W.), and the Departments of Surgery (J.A.L., T.K.H., T.M.) and Internal Medicine (G.G.S., D.C.B.), Washington University School of Medicine, St. Louis; and SangStat Medical Corporation, Menlo Park, Calif. (R.J.T.). Address reprint requests to Dr. Schnitzler at the Health Administration Program, Washington University School of Medicine, 4547 Clayton Ave., St. Louis, MO 63110, or at schnitz@wueconc.wustl.edu. 
Throughout the debate and study of this issue, little information has been available on the economic implications of HLA matching. Gjertson et al. argued that a savings of approximately $\$ 1,000$ per patient could be realized by five years after transplantation if cadaveric kidneys were allocated nationally on the basis of a hierarchical system designed to maximize HLA matches. ${ }^{1}$ However, their economic calculations were based on a model of the cost of return to dialysis, graft removal, and transportation of organs. Data on actual expenses for the care of transplant recipients were not available for their calculations.

We undertook an analysis of the actual medical expenses associated with different levels of HLA mismatching in cadaveric renal transplantation. These expenses include the cost of the transplantation, including the postoperative medical care and immunosuppression, and the cost of dialysis in patients whose grafts fail. Using UNOS registry data linked to Medicare claims and payment data, we calculated the average Medicare payments associated with various degrees of HLA mismatching between donor and recipient. Average Medicare payments associated with increasing cold-ischemia times were also calculated. These figures were combined in simulations to generate estimates of the economic ramifications of HLA-based methods of allocating cadaveric kidneys either locally or nationally.

\section{METHODS}

\section{Sources of Data}

All data were supplied by the U.S. Renal Data System (USRDS), ${ }^{6}$ a joint effort of the National Institute of Diabetes and Digestive and Kidney Diseases and the Health Care Financing Administration (HCFA). The system was designed to collect, analyze, and distribute descriptive data on end-stage renal disease in the United States, including prevalence, treatment methods, survival, and costs of care. The USRDS provided us with records for all transplantations performed in the United States and recorded in the UNOS renal-transplantation registry. The USRDS linked the UNOS registry to HCFA billing and payment records for the Medicare system. This linkage made possible the analysis both of the characteristics and outcomes of renal-transplant recipients and of the charges and payments for the medical services provided to them. Because 92.5 percent of all payments for medical services for patients with end-stage renal disease are from Medicare, payments from secondary insurance coverage, and patient copayments, ${ }^{7}$ it was possible to examine the clinical outcomes associated with characteristics of donors, recipients, and protocols for nearly every renal transplantation performed in the United States and to determine the actual costs to Medicare associated with the outcomes. Changes in organ-allocation policy would presumably affect all recipients of cadaveric organs, regardless of their health care coverage. Therefore, no exclusions based on health care coverage were made. The economic analysis presented here focuses on the expected effect of a change in allocation policy on average costs to Medicare for all transplantations of cadaveric kidneys in the United States.

\section{Determination of HLA Matching}

The algorithm we used to calculate the number of HLA mismatches is that currently used by UNOS to determine the extent of HLA mismatching for organ allocation. An HLA mismatch exists when a donor's HLA-A, B, or DR antigen does not match the recipient's corresponding HLA-A, B, or DR antigen. Numbers of HLA mismatches were calculated on the basis of UNOS coding.

\section{Theoretical Distribution of Organs}

Estimates of the local and national distributions of cadaveric kidneys for transplantation according to the number of HLA mismatches that might result from a system designed to ensure minimal HLA mismatching were obtained from Takemoto et al. ${ }^{3}$ These two simulated distributions of organs were chosen because the estimated national proportion of kidneys allocated to recipients with no HLA mismatches agreed with the figures reported by UNOS for cadaveric kidney transplantations in 1996. UNOS reported that 14 percent of cadaveric renal transplantations in 1996 involved no HLA mismatches, ${ }^{8}$ and Takemoto et al. estimated that a rate of 14 percent would result from a policy of national allocation. ${ }^{3}$

The simulated local distribution of organs was designed to represent allocation within an organ-procurement organization, which may cover an area from part of a large city to several states, and was based on a pool of 750 recipients. The pool for the simulated national distribution was made up of 5000 recipients. Although there are more than 40,000 patients on the waiting list for kidney transplants, ${ }^{8}$ a pool of 5000 was chosen, according to Takemoto et al., "to take into account the 'friction' in a national waiting list wherein selected recipients are often unavailable for transplant." 3

The simulations of Takemoto et al. showed the potential of a strategy of minimizing HLA mismatches at either the local or the national level to reduce the degree of mismatching from that which results from the current system. ${ }^{3}$ The current system of allocating kidneys grants points to a donor-recipient pair for certain combinations of two or fewer mismatches. ${ }^{8}$ Points are also granted for other factors. The organ is assigned to the donorrecipient pair with the most total points. The strategy of minimizing mismatching that we used in our simulations involves first identifying the set of recipients with the fewest HLA mismatches with a given donor organ. For example, for a given organ, the fewest mismatches possible might be three, and there might be several possible recipients for that organ with three mismatches. The organ would then be assigned to one of these recipients on the basis of the factors other than HLA matching that are currently used in the point system. The current system considers HLA matching and other factors simultaneously. By emphasizing mismatches before other factors and by taking into account more than two HLA mismatches, a system based on minimal HLA mismatching could much more effectively avoid transplantations with high numbers of mismatches.

According to current policy regarding local and national allocations, kidneys were assumed to be assigned to recipients with no HLA mismatches throughout the nation. The proportion of transplantations that involved donor-recipient pairs with no HLA mismatches was determined by actual experience with the current national allocation policy. We made adjustments to the estimates of local distribution made by Takemoto et al. so as to incorporate the current rate of transplantation of kidneys with no HLA mismatches (14 percent) in 1996. ${ }^{3,8}$

\section{Estimation of Costs}

Average cumulative costs were calculated according to a generalization of the Kaplan-Meier method for continuous data, ${ }^{9}$ an information-preserving method that incorporates data on patients with incomplete follow-up by systematically accounting for the censoring of observations at the time of the last known follow-up. The incremental addition to cumulative costs on any given day is the average cost on that day for the patients followed up to that point. For example, the addition to average cumulative costs on day 365 is simply the average of the costs incurred on day 365 by patients with 365 days of follow-up. Sequentially summing these average daily costs produces estimates of the average cost of care over time while conserving all available information. Patients were included in the analysis from the day of transplantation to the last follow-up contact or death. Therefore, this method includes all 
costs to Medicare of medical care - including, for example, hospitalization for transplantation, acute rejection, or infection; return to dialysis; and retransplantation.

\section{Statistical Analysis}

Measuring significance levels associated with average cumulative costs is difficult, because of the complex nature of the calculations involved. Mathematically derived standard errors are not available for the information measured in this case. Bootstrap methods produce very accurate estimates of error and confidence intervals by means of repeated random sampling from the original sample. ${ }^{10,11}$ Statistics of interest generated by the resampling provide information about the distribution of the variable being estimated. It is often suggested that 1000 bootstrap replications are the minimum necessary to produce confidence intervals. ${ }^{11}$ We performed 2000 bootstrap replications for each analysis.

Graft survival was estimated with the Kaplan-Meier method, with $\mathrm{P}$ values given for the log-rank test. ${ }^{9}$ Differences were considered significant at an alpha level of 0.05 . All tests were two-tailed.

\section{RESULTS}

\section{Patients}

All patients recorded in the UNOS registry (distributed with the 1999 release of the USRDS data base $^{6}$ ) as undergoing renal transplantation from 1991 through 1997 were initially included in the analysis. We excluded recipients of transplants from living donors, those who received multiorgan transplants, and those with missing information on HLA matching. Patients who received first transplants before 1991 were also excluded. A total of 27,050 patients remained in the analysis of the effects of HLA matching. Among these patients, the duration of cold ischemia was recorded for 26,977 .

\section{HLA Matching}

Average cumulative Medicare payments for patients receiving transplants with zero to six HLA mismatches are shown in Figure 1. As expected, transplantations involving no HLA mismatches were associated with the lowest average Medicare payments $(\$ 60,436$ at three years), and those involving six HLA mismatches with the highest payments $(\$ 80,807$ at three years, $\mathrm{P}<0.001)$. Average Medicare payments through three years after transplantation and three-year graft-survival rates are presented in Table 1 . From zero to six HLA mismatches, the total cost increased by $\$ 20,371$, or 34 percent.

The reduction in the graft-survival rate from transplantations involving no HLA mismatches to those involving six was 11 percent $(\mathrm{P}<0.001)$. The most pronounced effect of increased HLA mismatching on graft survival, a decrease of 4 percent, occurred from zero to one mismatch $(\mathrm{P}<0.001)$. However, the most pronounced economic effect of increased HLA mismatching was the 12 percent increase from five to six mismatches, representing an increase of $\$ 8,692$ in cost $(\mathrm{P}<0.001)$. The difference in cost between five and six HLA mismatches accounts for 43 percent of the total difference between zero and six mismatches.

The percentages of transplantations involving var- ious numbers of HLA mismatches are shown in Table 1 . The actual distribution of numbers of HLA mismatches is that reported by UNOS for $1996 .{ }^{8}$ The simulated local system for the allocation of organs shifted the number of mismatches involved in most transplantations from four, five, or six to one or two. The larger pool of recipients used for the simulated national allocation system generated further improvement in the distribution of HLA mismatches; the result was more transplantations involving one mismatch and fewer involving two or three mismatches, as compared with the simulated local distribution.

The average expected cost savings from a policy ensuring that transplantations involved the fewest possible HLA mismatches, assuming no effects due to differences in cold-ischemia time, was calculated as the difference between the weighted average cost of transplantations according to the actual distribution and that according to the simulated distributions. The average expected cost savings amounted to $\$ 4,290$ for the simulated local distribution and $\$ 4,768$ for the simulated national distribution. Similarly, the average expected increase in the rate of graft survival, assuming no effects due to differences in cold-ischemia time, was 2.3 percent for the simulated local distribution and 2.7 percent for the simulated national distribution.

\section{Duration of Cold Ischemia}

Average cumulative Medicare payments through three years after transplantation are shown in Figure 2 according to the duration of cold ischemia. A regular increase in cost is apparent as cold-ischemia time increases. By three years after transplantation, the average Medicare payments were $\$ 64,119$ for transplantations involving less than 12 hours of cold ischemia and $\$ 74,997$ for those involving more than 36 hours of cold ischemia $(\mathrm{P}<0.001)$. The economic effect of the duration of cold ischemia was similar for transplantations involving different levels of HLA matching, including those with no HLA mismatches.

The effects of the duration of cold ischemia on cost and graft survival at three years are presented in Table 2. To calculate the average increase in Medicare payments for an increase of one hour in coldischemia time, the difference in average Medicare payments from one group of patients to another was divided by the difference in the average number of hours of cold ischemia. On the basis of these calculations, we then determined that the weighted average cost associated with an increase of one hour of cold ischemia through three years after transplantation was \$290. Thus, an additional 10 hours of cold ischemia would be expected to raise the average cost of transplantation at three years by $\$ 2,900$. On the basis of weighted averages, the effect of an increase of one hour of cold ischemia was a reduction of 0.3 percent in graft survival at three years. 


\section{Comparisons of Allocation Schemes}

The implications of the allocation of cadaveric renal kidneys according to the methods used in our simulations, in terms of average graft-survival rates and average cost savings to Medicare, are shown in Table 3 . The effects of such a system of allocation include both the potential benefits of reallocation in terms of better HLA matching and the additional coldischemia time necessitated by shipping kidneys to other regions of the country. The actual average recorded duration of cold ischemia for kidneys transplanted locally was 19 hours, as compared with 24 hours for kidneys transported on the basis of national allocation. The calculations of the potential effects of a national allocation system were based on the assumption that an average of five additional hours of cold ischemia would be required. Graft survival and cost with the national allocation scheme were also calculated for 0 and 10 additional hours of cold ischemia for comparative purposes. The potential effects of local allocation according to a system designed to minimize HLA mismatches were calculated, on the assumption that there would be no increase in cold ischemia. Although it is unlikely that reallocation at the local level would increase cold-ischemia time for the average transplantation, cost calculations were also made with one and two additional hours assumed, for comparative purposes. The results were calculated as the potential savings associated with a given allocation system minus the number of additional hours of cold ischemia multiplied by the average cost of one additional hour.

The results of our calculations suggest that a strategy of national allocation of cadaveric kidneys that was designed to minimize HLA mismatching would generate the largest improvement in graft survival and the largest cost savings if there were no effects on the duration of cold ischemia. However, longer preservation times would be necessary under a national allocation policy. Our model predicted that a policy of local allocation aimed at ensuring minimal HLA mismatching would generate the greatest improvement in graft survival and the greatest cost savings once cold-ischemia time was taken into account; the average graft-survival rate would increase by 2.3 percent, with average cost savings of $\$ 4,290$ by three years

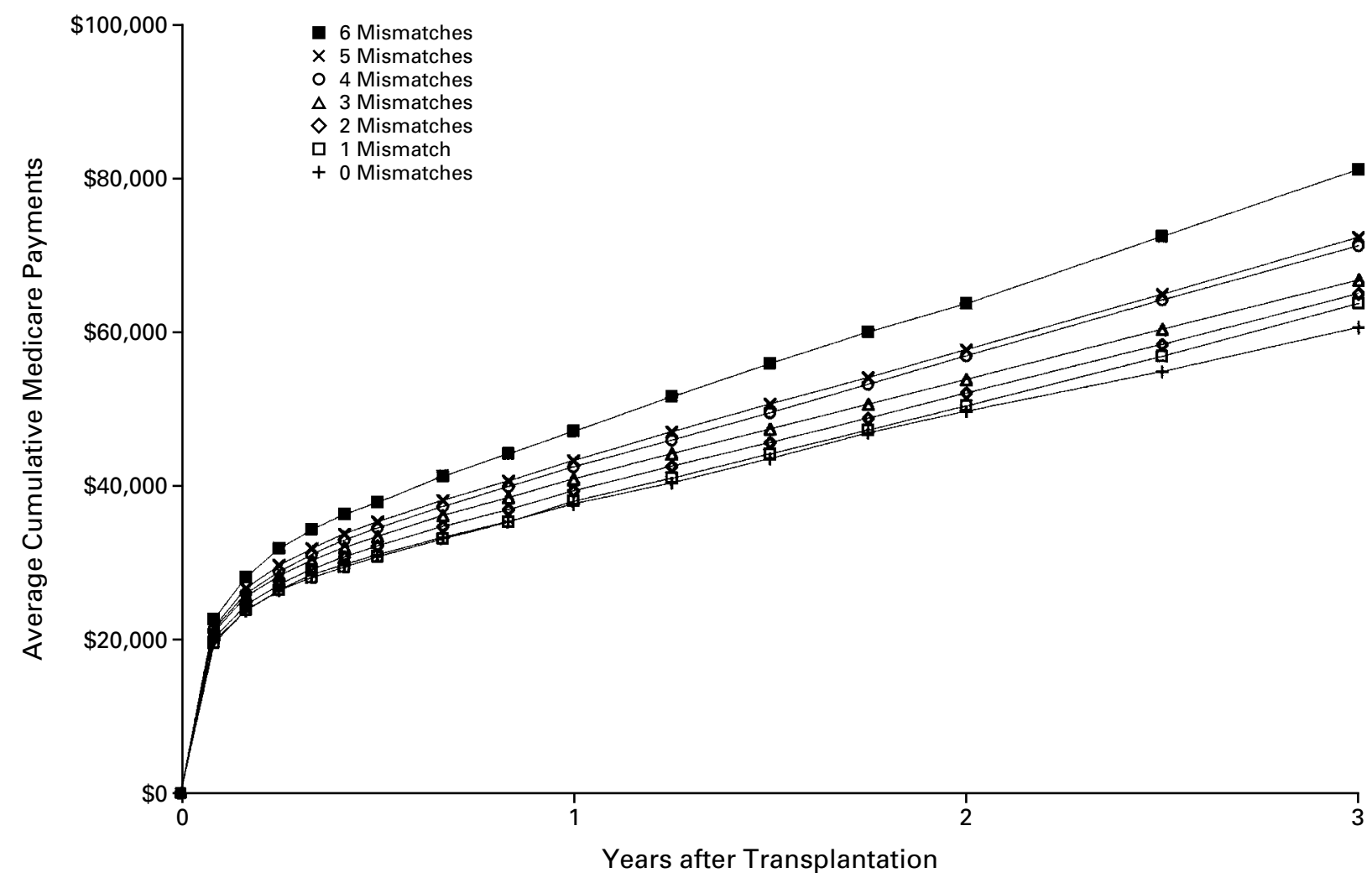

Figure 1. Average Cumulative Medicare Payments for Patients Receiving Cadaveric Renal Transplants, According to the Number of HLA Mismatches.

The absence of HLA mismatches was associated with the lowest average Medicare payments (\$60,436 at three years), and six HLA mismatches were associated with the highest average Medicare payments $(\$ 80,807$ at three years, $P<0.001$ for the comparison between zero and six mismatches). 
Table 1. Actual and Theoretical Proportions of Transplantations Involving Various Numbers of HLa Mismatches, Graft-Survival Rates, and Average Medicare Payments at Three Years after Transplantation. *

\begin{tabular}{|c|c|c|c|c|c|}
\hline \multirow[t]{3}{*}{$\begin{array}{l}\text { No. OF HLA } \\
\text { MisMATCHES }\end{array}$} & \multicolumn{3}{|c|}{$\begin{array}{l}\text { DisTRIBUTIONS OF HLA } \\
\text { MISMATCHEST }\end{array}$} & \multirow[t]{2}{*}{$\begin{array}{l}\text { Medicare } \\
\text { PaYMENTS }\end{array}$} & \multirow[t]{2}{*}{$\begin{array}{c}\text { Graft } \\
\text { SuRVIVAL }\end{array}$} \\
\hline & $\begin{array}{c}\text { ACTUAL } \\
1996\end{array}$ & $\begin{array}{l}\text { SIMULATED } \\
\text { LOCAL } \\
\text { SYSTEM }\end{array}$ & $\begin{array}{l}\text { SIMULATED } \\
\text { NATIONAL } \\
\text { SYSTEM }\end{array}$ & & \\
\hline & & percent & & dollars & percent \\
\hline 0 & 14 & 14 & 14 & $60,436 \pm 756$ & $83 \pm 1.0$ \\
\hline 1 & 3 & 24 & 45 & $63,569 \pm 1,044$ & $79 \pm 1.0$ \\
\hline 2 & 11 & 44 & 35 & $64,853 \pm 565$ & $78 \pm 0.7$ \\
\hline 3 & 23 & 18 & 5 & $66,584 \pm 417$ & $77 \pm 0.6$ \\
\hline 4 & 25 & 1 & 1 & $71,031 \pm 461$ & $74 \pm 0.5$ \\
\hline 5 & 16 & 0 & 0 & $72,115 \pm 571$ & $74 \pm 0.6$ \\
\hline 6 & 8 & 0 & 0 & $80,807 \pm 1,061$ & $72 \pm 1.0$ \\
\hline
\end{tabular}

*Plus-minus values are means \pm SE. Because of rounding, percentages do not always total 100 .

†Actual 1996 data are from UNOS. ${ }^{8}$ Data from simulated local and national allocation systems are based on a model in which it was assumed that there was no effect of cold ischemia and that HLA mismatching was minimized. after transplantation. National allocation according to this strategy would improve average graft survival by 1.2 percent and would save Medicare an average of $\$ 3,318$ per transplant recipient by three years after transplantation, given the expected increase of five hours in the duration of cold ischemia. For purposes of comparison, the local system of allocation with minimal HLA mismatching would produce results similar to those of the national scheme if the local policy added an average of $31 / 2$ hours to the duration of cold ischemia.

\section{DISCUSSION}

The results of our simulations suggest that the extent of HLA mismatching is an important determinant of Medicare expenditures associated with renal transplantation. Much like the effect of HLA mismatching on graft survival, ${ }^{4}$ the effect of HLA mismatching on costs is not constant over the range from zero to six mismatches. The benefit of reduced HLA mismatching, in terms of graft survival, is most pronounced when there are no HLA-antigen mismatches; however, the effect of mismatching on costs

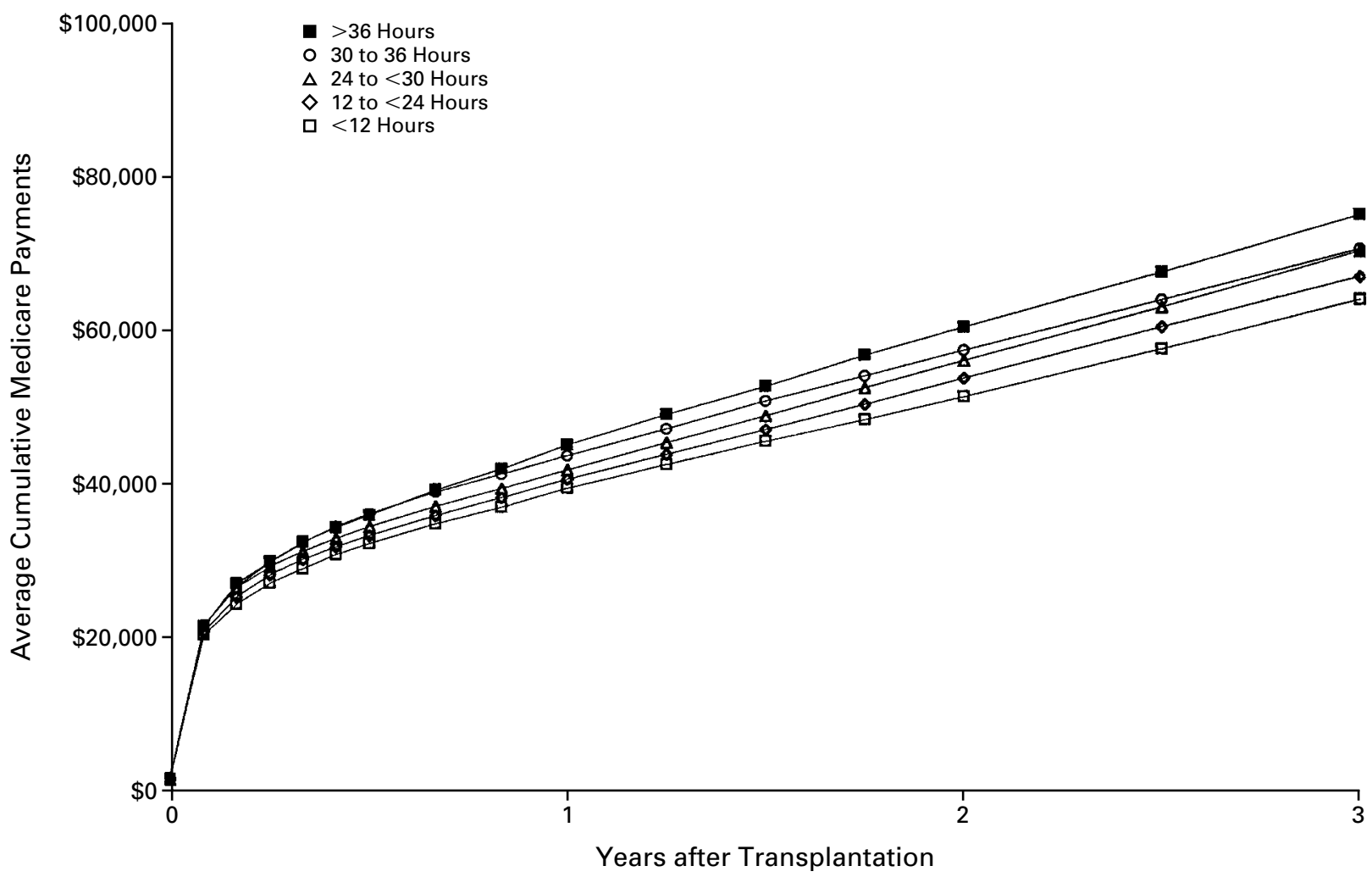

Figure 2. Average Cumulative Medicare Payments for Patients Receiving Cadaveric Renal Transplants, According to the Duration of Cold Ischemia.

By three years after transplantation, the average Medicare payments were $\$ 64,119$ for transplantations involving less than 12 hours of cold ischemia and $\$ 74,997$ for those involving more than 36 hours of cold ischemia $(P<0.001)$. 
Table 2. Duration of Cold Ischemia, Average Medicare Payments, and Graft Survival Rates at Three Years after Transplantation.*

\begin{tabular}{|c|c|c|c|c|c|}
\hline $\begin{array}{l}\text { DuRation } \\
\text { OF Cold } \\
\text { IsCHEMIA }\end{array}$ & $\begin{array}{c}\text { PERCENT } \\
\text { OF } \\
\text { TrANS- } \\
\text { PLANTS }\end{array}$ & $\begin{array}{l}\text { AvERAGE } \\
\text { COLD- } \\
\text { ISCHEMIA } \\
\text { TIME }\end{array}$ & $\begin{array}{l}\text { Average } \\
\text { MEDICARE } \\
\text { PaymENTS }\end{array}$ & $\begin{array}{c}\text { AvERAGE } \\
\text { INCREASE IN } \\
\text { PAYMENTS/HR }\end{array}$ & $\begin{array}{c}\text { Graft } \\
\text { SuRVIVAL }\end{array}$ \\
\hline & & $\mathrm{hr}$ & \multicolumn{2}{|c|}{ dollars } & $\%$ \\
\hline$<12 \mathrm{hr}$ & 11 & 8 & $64,119 \pm 660$ & - & $78 \pm 0.5$ \\
\hline 12 to $<24 \mathrm{hr}$ & 49 & 18 & $67,054 \pm 311$ & 294 & $76 \pm 0.3$ \\
\hline 24 to $<30 \mathrm{hr}$ & 20 & 27 & $70,421 \pm 508$ & 374 & $74 \pm 0.6$ \\
\hline 30 to $36 \mathrm{hr}$ & 12 & 33 & $70,638 \pm 680$ & 43 & $74 \pm 0.8$ \\
\hline$>36 \mathrm{hr}$ & 8 & 43 & $74,997 \pm 852$ & 436 & $70 \pm 1.0$ \\
\hline
\end{tabular}

*Plus-minus values are means \pm SE.

Table 3. Predicted Cost Savings to Medicare and Changes in Graft Survival with Revised National and Local Allocation Systems Based on Minimal HLa Mismatching, as Compared with the Current Allocation System.*

\begin{tabular}{cccc}
\hline $\begin{array}{c}\text { Revised } \\
\text { Allocation } \\
\text { System }\end{array}$ & $\begin{array}{c}\text { Average } \\
\text { INCREASE IN } \\
\text { Cold Ischemia }\end{array}$ & $\begin{array}{c}\text { Average } \\
\text { Change IN } \\
\text { Graft Survivalt }\end{array}$ & $\begin{array}{c}\text { Average } \\
\text { DeCREASE IN } \\
\text { Total Payments }\end{array}$ \\
National & $\mathrm{hr}$ & $\%$ & dollars \\
& 0 & 2.7 & 4,768 \\
Local & 5 & 1.2 & 3,318 \\
& 10 & -0.3 & 1,868 \\
& 0 & 2.3 & 4,290 \\
& 1 & 2.0 & 4,000 \\
\hline
\end{tabular}

*Data for the current system are from 1996. Costs and graft-survival rates are at three years.

†The negative number indicates a decrease in graft survival; others indicate increases.

is most pronounced when there are six mismatches. The economic effect of using kidneys with six as compared with five HLA mismatches accounts for 43 percent of the total difference in cost between transplantations involving zero mismatches and those involving six mismatches.

Although there is a clear potential to save money through better HLA matching, higher costs are also associated with increasing cold-ischemia time. The costs associated with longer preservation limit the likelihood that any national scheme for the allocation of cadaveric kidneys can realize the full benefits of better HLA matching. Although cost savings and better graft survival may result from any move to improve the degree of HLA matching, better local matching (within organ-procurement organizations) is likely to have better results than a national alloca- tion scheme. Our simulations demonstrate that better matching at the local level would produce the largest cost savings and the greatest benefit in terms of graft survival when cold-ischemia time is taken into account. This conclusion is further supported by the fact that organizational and organ-transportation costs were excluded from the analysis presented here, which focused on direct medical costs. Gjertson et al. ${ }^{1}$ assumed that organizational and shipping costs in a national system of allocation would average approximately $\$ 1,000$ per transplantation. ${ }^{1}$ The inclusion of estimated shipping costs in our calculations would have reduced further the relative economic benefit of national, as compared with local, allocation. Although the actual magnitude of these costs is uncertain, they would have to be considered before a national allocation system could be implemented.

The cost associated with longer cold ischemia times was a critical factor in this analysis. Ten hours of cold ischemia costs Medicare an average of $\$ 2,900$ and reduces graft-survival rates by 3 percent at three years after transplantation. This observation has important implications for health care policy. Is it necessary to have average cold-ischemia times of 19 hours for transplants locally distributed and 24 hours for those shared nationally? Numerous strategies might be used to reduce the length of preservation, such as giving transplantation procedures priority in the assignment of operating rooms and other hospital resources and using chartered flights when organs are shipped. Approximately 8000 transplantations of cadaveric kidneys are performed each year in the United States; the annual savings to Medicare from every hour by which the average cold-ischemia time is reduced is therefore approximately $\$ 2.3$ million.

The economic benefits of the local allocation of kidneys according to a system designed to minimize HLA mismatching result predominantly from avoiding transplantation involving the highest numbers of mismatches. By far the most expensive transplantations are those with six HLA mismatches, but transplantations involving four or five HLA mismatches are also markedly more expensive than those involving three or fewer HLA mismatches. Much of the economic benefit of a system of local allocation designed to minimize HLA mismatching could be achieved by a policy that limited transplantations of highly mismatched grafts.

Numerous issues remain to be addressed. If a redesign of the organ-allocation system is to be considered, it is important to consider equity in the resulting distribution of organs. Adjustments to the present scheme may alter the degree of preference currently given to children, highly immune-sensitized patients, patients with long waiting times, and patients who have served as donors for others. Excessive focus on HLA matching has been implicated in the apparent racial inequities in the current distribution of organs. ${ }^{5}$ 
Another issue to be considered is who is to benefit from any cost savings that are generated. Considering that 8000 renal transplantations are performed each year in the United States, a local system of allocation of kidneys to ensure minimal HLA mismatching has the potential to save Medicare a total of \$34 million through three years after transplantation. Our model projects that this sum will increase in later years. One potential use of the money saved would be to cover the cost of maintenance immunosuppression. Medicare coverage of immunosuppression ends three years after transplantation. If a new organ-allocation policy is implemented, a portion of any savings that is generated can be used to extend the duration of this coverage.

For many reasons, policy makers are moving toward a more national scheme of allocation. In this time of increasing financial pressure on our medical system, such a redesign should be undertaken with the understanding that - because of the detrimental effects of the longer cold-ischemia time that is likely in a national allocation scheme - the economic benefits resulting from greater HLA matching are most readily available at the local level. Ignoring this fact may make an already imperfect system worse.

We are indebted to Ray $R$. White, who provided the code on which the HLA-matching algorithm was based; to Ann Harper, who provided documentation of UNOS policy; and to other members of the
UNOS staff, without whose assistance this study would not have been possible. The calculations and opinions in this article are those of the authors, and not of UNOS or the UNOS staff. In addition, the interpretation and reporting of the data supplied by the USRDS are the responsibility of the authors and should in no way be seen as reflecting the official policy or interpretation of the U.S. government.

\section{REFERENCES}

1. Gjertson DW, Terasaki PI, Takemoto S, Mickey MR. National allocation of cadaveric kidneys by HLA matching: projected effect on outcome and costs. N Engl J Med 1991;324:1032-6.

2. Takemoto S, Terasaki PI, Cecka JM, Cho YW, Gjertson DW. Survival of nationally shared, HLA-matched kidney transplants from cadaveric donors. N Engl J Med 1992;327:834-9.

3. Takemoto S, Gjertson DW, Terasaki PI. HLA matching: maximizing the number of compatible transplants. In: Terasaki PI, Cecka M, eds. Clinical transplants 1993. Los Angeles: UCLA Tissue Typing Laboratory, 1994: 521-31.

4. Held PJ, Kahan BD, Hunsicker LG, et al. The impact of HLA mismatches on the survival of first cadaveric kidney transplants. N Engl J Med 1994;331:765-70.

5. Gaston RS, Ayres I, Dooley LG, Diethelm AG. Racial equity in renal transplantation: the disparate impact of HLA-based allocation. JAMA 1993;270:1352-6.

6. Renal Data System. Researchers' guide to the USRDS database. Bethesda, Md.: National Institute of Diabetes and Digestive and Kidney Diseases, 1998.

7. Idem. USRDS 1999 annual report. Bethesda, Md.: National Institute of Diabetes and Digestive and Kidney Diseases, 1999.

8. 1997 Annual report of the U.S. Scientific Registry of Transplant Recip ients and the Organ Procurement and Transplantation Network: transplant data: 1988-1996. Richmond, Va.: United Network for Organ Sharing, 1997.

9. Kaplan EL, Meier P. Nonparametric estimation from incomplete observations. J Am Stat Assoc 1958;53:457-81.

10. Efron B. The jackknife, the bootstrap, and other resampling plans. Philadelphia: Society for Industrial and Applied Mathematics, 1982.

11. Hollander M, Wolfe DA. Nonparametric statistical methods. 2nd ed. New York: John Wiley, 1999. 